\title{
Association of napping with incident cardiovascular events in a prospective cohort study
}

\author{
Nadine Häusler ำ , ${ }^{1}$ Jose Haba-Rubio, ${ }^{2}$ Raphael Heinzer, ${ }^{2}$ Pedro Marques-Vidal ${ }^{1}$
}

- Additional material is published online only. To view please visit the journal online (http://dx.doi.org/10.1136/ heartjnl-2019-314999).

'Department of Medicine, Internal Medicine, University Hospital of Lausanne, Lausanne, Switzerland

${ }^{2}$ Centre for Investigation and Research in Sleep (CIRS), University Hospital of Lausanne, Lausanne, Switzerland

Correspondence to Dr Nadine Häusler, Department of Medicine, Internal Medicine, University Hospital of Lausanne, Lausanne 1011, Switzerland; Nadine.Hausler@chuv.ch

$J H-R, R H$ and PM-V contributed equally.

Received 2 March 2019 Revised 18 June 2019 Accepted 25 June 2019 Published Online First 9 September 2019

\section{SLinked}

- http://dx.doi.org/10.1136/ heartjnl-2019-315442

Check for updates

(c) Author(s) (or their employer(s)) 2019. No commercial re-use. See rights and permissions. Published by BMJ.

To cite: Häusler N, HabaRubio J, Heinzer R, et al. Heart 2019;105:1793-1798.

\begin{abstract}
Objective There is controversy regarding the effect of napping on cardiovascular disease (CVD), with most studies failing to consider napping frequency. We aimed to assess the relationship of napping frequency and average nap duration with fatal and non-fatal CVD events.
\end{abstract}

Methods 3462 subjects of a Swiss population based cohort with no previous history of CVD reported their nap frequency and daily nap duration over a week, and were followed over 5.3 years. Fatal and nonfatal CVD events were adjudicated. Cox regressions were performed to obtain HRs adjusted for major cardiovascular risk factors and excessive daytime sleepiness or obstructive sleep apnoea.

Results 155 fatal and non-fatal events occurred. We observed a significantly lower risk for subjects napping 1-2 times weekly for developing a CVD event (HR $0.52,95 \% \mathrm{Cl} 0.28$ to 0.95 ) compared with nonnapping subjects, in unadjusted as well as adjusted models. The increased HR $(1.67,95 \% \mathrm{CI} 1.10$ to 2.55$)$ for subjects napping 6-7 times weekly disappeared in adjusted models (HR $0.89,95 \% \mathrm{Cl} 0.58$ to 1.38). Neither obstructive sleep apnoea nor excessive daytime sleepiness modified this lower risk. No association was found between nap duration and CVD events.

Conclusion Subjects who nap once or twice per week have a lower risk of incident CVD events, while no association was found for more frequent napping or napping duration. Nap frequency may help explain the discrepant findings regarding the association between napping and CVD events.

\section{INTRODUCTION}

Habitual daytime napping is a common practice worldwide. $^{12}$ Whereas daytime napping has been consistently linked to overall mortality, ${ }^{1-3}$ the effect of napping on cardiovascular disease (CVD) remains unclear. A Greek cohort study found that habitual nappers have lower coronary mortality, ${ }^{4}$ and a Greek case control study found that nappers have a lower risk of coronary heart disease. ${ }^{5}$ In contrast, a case control study in Costa Rica, ${ }^{6}$ and three cohort studies in the USA, ${ }^{7}$ Japan $^{8}$ and Germany, ${ }^{9}$ all reported increased risk either for cardiac events $^{69}$ or CVD mortality. ${ }^{78}$ Other studies found no association between habitual daytime napping and CVD. ${ }^{1-310}$ Only a few studies considered nap frequency and investigated the effect of irregular napping on CVD. Whereas the Greek cohort study found that irregular nappers (taking naps once or twice per week) had lower coronary mortality, ${ }^{4}$ no relationship between irregular nappers $(<5$ naps weekly) and cardiovascular mortality was found in the German cohort study. ${ }^{9}$

Nap duration was reported to be important with respect to CVD. A meta-analysis found a J curved dose-response relationship between nap duration and CVD. ${ }^{11}$ They further reported that subjects taking long naps ( $\geq 1$ hour) had a higher risk for CVD compared with subjects taking no naps, while no association between short naps ( $<1$ hour) and CVD was found. ${ }^{11}$ Interestingly, a J curved relationship was also reported between nap duration and diabetes and metabolic syndrome. ${ }^{12}$ A large study $(n=116632)$ reported that, compared with nonnappers, the risk of CVD increased for subjects napping $\leq 1$ hour and was highest for subjects napping $>1$ hour. ${ }^{13}$ In contrast, in the Greek cohort study, a lower risk for coronary mortality was found for subjects taking naps of any duration. ${ }^{4}$ Apart from nap duration, night time sleep duration in combination with napping seems to play a role with respect to CVD. A study showed that daytime napping is associated with an increased risk of major CVD events in subjects with $>6$ hours of night time sleep but not in subjects sleeping $\leq 6$ hours/night. ${ }^{13}$ Contrarily, habitual nappers sleeping $<6$ hours/ night were at higher risk of CVD mortality than habitual nappers sleeping 6-8 hours/night. ${ }^{7}$

Most studies only distinguished between nappers and non-nappers ${ }^{1-3} 5-810$ or investigated the effect of nap duration ${ }^{491113}$ on CVD, but did not take the frequency of napping into account. Further, most studies only focused on CVD mortality, and failed to assess the effect of napping on non-fatal CVD events. Thus we aimed to investigate the effect of nap frequency and average daily nap duration over a week on fatal and non-fatal CVD events in a population based cohort.

\section{METHODS \\ Study population}

CoLaus is a Swiss population based, observational, prospective cohort study investigating determinants of CVD. ${ }^{14}$ Between 2003 and 2006, a random sample of 6733 subjects (age range 35-75 years, $52.5 \%$ women) living in the city of Lausanne, Switzerland (117161 habitants) was drawn. ${ }^{14}$ The first follow-up was conducted between April 2009 and September 2012 and included 5064 subjects; the second follow-up was conducted between May 2014 and April 2017 (median follow-up time 5.3 years after the first follow-up). As sleep and nap data were collected at the first follow-up onwards, 
the first follow-up will thereafter be designated the 'baseline', and the second follow-up as the 'follow-up'.

The institutional ethics committee of the University of Lausanne, which afterwards became the Ethics Commission of Canton Vaud (www.cer-vd.ch) approved the CoLaus study (reference $16 / 03$, decisions on 13 January and 10 February 2003) and the approval was renewed for the follow-ups (reference 33/09, decision 23 February 2009; reference 26/14, decision 11 March 2014). The study complies with the Declaration of Helsinki and written informed consent was obtained from all participants.

\section{Nap occurrence, frequency and duration}

Naps were assessed by the validated Physical Activity Frequency Questionnaire ${ }^{14}$ where subjects self-reported the frequency and duration of 70 types of activities (including napping) during the previous week. Napping was assessed by the item 'Sieste ou repos au lit l'après-midi' (nap or bed rest in the afternoon). Subjects were considered as nappers (as opposed to non-nappers) when they reported to have napped at least once during the previous week. Napping frequency was categorised into non-nappers, 1-2 naps, 3-5 naps and 6-7 naps during the previous week. Nap duration was multiplied by how often subjects reported to be napping over a week and divided by 7 . In order to be consistent with previous studies, ${ }^{11} 13$ we dichotomised the daily nap duration over a week into $<1$ hour and $\geq 1$ hour.

\section{Sleep data}

Sleep duration was assessed by the Pittsburgh Sleep Quality Index, ${ }^{15}$ in which subjects reported their average hours of sleep per night during the last month. Daytime sleepiness was assessed using the Epworth sleepiness scale ${ }^{16}$ and excessive daytime sleepiness was considered for a sum score $\geq 11$.

Polysomnography was performed on a subsample of the cohort (42.7\%), as described previously. ${ }^{17}$ The severity of obstructive sleep apnoea (OSA) was measured by the apnoeahypopnoea index (AHI) and categorised as none (AHI <5/ hour), mild ( $5 \leq \mathrm{AHI}<15$ events per hour of sleep), moderate (15 $\leq$ AHI $<30$ events per hour of sleep) and severe (AHI $\geq 30$ events per hour of sleep).

\section{Collection and adjudication of CVD events}

All fatal and non-fatal CVD events occurring between baseline and follow-up were adjudicated by a panel of independent experts, and the detailed procedure is described in the online supplementary file 1 . For this study, we used a composite endpoint grouping all fatal and non-fatal CVD events (acute coronary syndromes and strokes, ischaemic heart disease). In the case of multiple CVD events, only the first one was considered in the analysis.

\section{Covariates}

Participants were invited to attend the outpatient clinic at the University Hospital of Lausanne, Switzerland, in the morning after overnight fasting for clinical assessment, blood sample collection and questionnaire completion. Body weight and height were measured using a calibrated scale and a vertical stadiometer, respectively (Seca, Hamburg, Germany). Body mass index (BMI) was calculated as weight in $\mathrm{kg}$ divided by the square of the participant's height in metres: overweight and obesity were defined as BMI $\geq 25-30 \mathrm{~kg} / \mathrm{m}^{2}$ and $\geq 30 \mathrm{~kg} / \mathrm{m}^{2}$, respectively. Systolic and diastolic blood pressures were evaluated in triplicate on the left arm at $5 \mathrm{~min}$ intervals with the participant seated and resting for at least $10 \mathrm{~min}$ using a calibrated automated oscillometric sphygmomanometer (Omron HEM-907, Matsusaka, Japan), ${ }^{18}$ and the average of the last two measurements was considered for analysis. Hypertension was defined as systolic blood pressure $\geq 140 \mathrm{~mm} \mathrm{Hg}$ or diastolic blood pressure $\geq 90 \mathrm{~mm} \mathrm{Hg}$, or current antihypertensive treatment. Glucose was quantified by colorimetric assays as previously described. ${ }^{14}$ Diabetes was defined as a fasting blood glucose level of $\geq 7 \mathrm{mmol} / \mathrm{L}$ or current antidiabetic treatment. Dyslipidaemia was defined as low density liporprotein cholesterol $>4.9 \mathrm{mmol} / \mathrm{L}$ or current hypolipidaemic treatment. Depressive status was measured with the previously validated 20 item Centre for Epidemiologic Studies-Depression Scale questionnaire, and depressive status was considered for a score of at least 17 for men and 23 for women. ${ }^{19}$

Age, sex, educational attainment (low, middle, high) and smoking status (current, former/never) were assessed by questionnaire. Sedentary behaviour was assessed by the Physical Activity Frequency Questionnaire ${ }^{20}$ and defined as spending $>90 \%$ of daily energy in activities below moderate and high intensity. ${ }^{21}$

\section{Statistical analyses}

Statistical analyses were performed using STATA 15.1 (StataCorp, College Station, Texas, USA). Continuous variables are summarised as mean $\pm S D$ and categorical variables as number of subjects (\%). The Pearson $\chi^{2}$ test or ANOVA was used to associate sleep variability measures and obesity, diabetes and hypertension. We analysed the effect of napping, nap frequency and daily nap duration over a week on CVD. Cox regressions were performed and HRs and 95\% CIs obtained for: (1) crude model; (2) model 2, adjusting for age, sex, education (low, middle, high), smoking status (current, former, never), sedentary behaviour (yes/no), BMI (normal, overweight, obese) and sleep duration (continuous); and (3) model 3, with further adjusting for hypertension, diabetes and dyslipidaemia (categorised as yes/ no).

Several sensitivity analyses were performed on the fully adjusted model (model 3). First, we included severity of OSA as a covariate, as subjects with OSA might compensate impaired sleep quality with naps. Second, we included excessive daytime sleepiness as a covariate, as excessive daytime sleepiness has been shown to be associated with both naps ${ }^{22}$ and CVD. ${ }^{23}$ Third, we excluded subjects $<65$ years as they might be refrained from napping due to their employment. Fourth, we excluded nonnappers. Fifth, we stratified our analyses based on nocturnal sleep duration ( $<6$ hours/ $\geq 6$ hours). However, it was not possible

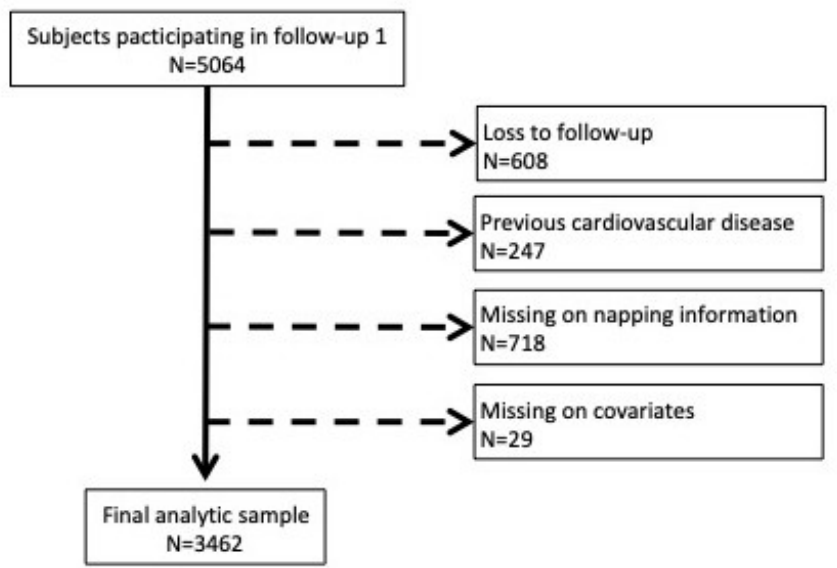

Figure 1 Exclusion procedure, CoLaus study, Lausanne, Switzerland, 2009-2017. 
to perform the sensitivity analyses for subjects with $<6$ hours of sleep duration due to the low numbers which resulted in an empty cell (see online supplementary table 1). Sixth, we additionally adjusted for depressive status, as depression is a potential confounder in the relationship between napping and CVD events. $^{24}$

\section{Exclusion criteria}

Subjects were excluded if they: (1) were lost to follow-up, (2) had a previous history of CVD, (3) had missing information on napping or (4) had missing covariates.

\section{RESULTS}

\section{Selection of subjects}

Of the 5064 subjects participating in the first follow-up, 608 were excluded due to lost to follow-up, 247 had a previous history of CVD, 718 had missing information on napping and 29 had missing information on covariates, resulting in a study sample of 3462 subjects (68.4\%) (figure 1). Excluded subjects were older, more frequently men, with a lower educational status, more frequently smokers, with a higher BMI, with higher average sleep duration and were more frequently nappers than the included subjects (see online supplementary table 2).

\section{Prevalence and characteristics of nappers}

Whereas most subjects reported no napping during the previous week ( $\mathrm{n}=2014,58 \%), 667$ subjects reported taking 1-2 naps (19\%), 411 subjects reported taking 3-5 naps (12\%) and 370 subjects reported taking 6-7 naps (11\%) during the previous week (table 1). Frequent nappers (ie, 3-7 times weekly) were older, more frequently men, with a lower educational status, were more frequently smokers, had a higher BMI and had a longer nocturnal sleep duration compared with non-nappers. Frequent nappers had more frequently excessive daytime sleepiness and had more severe OSA compared with non-nappers. We found a J curved relationship between nap frequency and CVD events (table 2). The highest percentage of subjects developing an event was found in subjects taking 6-7 naps weekly, and the lowest percentage was found among subjects taking 1-2 naps weekly.

\section{Naps and incidence of CVD events}

During the 17976 person-year follow-up (median follow-up 5.3 years, last exit 7.8 years), 155 fatal and non-fatal CVD events occurred.

In the crude model, subjects taking 1-2 naps weekly had a lower $\operatorname{HR}(0.39,95 \% \mathrm{CI} 0.21$ to 0.72$)$ and subjects taking 6-7 naps weekly had a higher HR $(1.67,1.10-2.55)$ for CVD events compared with non-nappers (table 3 ). The association between taking 1-2 naps weekly and lower incidence of CVD events remained after adjusting for age, sex, education, smoking status, sedentary behaviour, BMI and sleep duration $(0.52,0.28-0.92)$, and for cardiovascular risk factors $(0.52,0.28-0.95)$. This is also reflected in the highest survival probability for subjects napping 1-2 times weekly (figure 2). In contrast, no association was

Table 1 Characteristics of study sample according to nap frequency, CoLaus study, Lausanne, Switzerland ( $n=3462)$

\begin{tabular}{|c|c|c|c|c|c|}
\hline & \multirow[b]{2}{*}{ No nap } & \multicolumn{3}{|c|}{ Nap frequency } & \multirow[b]{2}{*}{$P$ value } \\
\hline & & 1-2 weekly & 3-5 weekly & 6-7 weekly & \\
\hline No $(\%)$ & $2014(58.1)$ & $667(19.3)$ & $411(11.9)$ & $370(10.7)$ & \\
\hline Age (years) $($ mean $\pm S D)$ & $56.5(10.2)$ & $53.9(9.1)$ & $59.6(9.9)$ & $63.1(10.3)$ & $<0.001$ \\
\hline $\operatorname{Men}(n(\%))$ & $843(41.9)$ & $303(45.4)$ & $205(49.9)$ & $208(56.2)$ & $<0.001$ \\
\hline Educational status (n (\%)) & & & & & $<0.001$ \\
\hline High & $472(23.4)$ & $195(29.2)$ & $87(21.2)$ & $49(13.2)$ & \\
\hline Middle & $536(26.6)$ & $195(29.2)$ & $125(30.4)$ & $84(22.7)$ & \\
\hline Low & $1006(50.0)$ & $277(41.5)$ & $199(48.4)$ & $237(64.1)$ & \\
\hline Smoker (n (\%)) & $372(18.5)$ & $164(24.6)$ & $82(20.0)$ & $73(19.7)$ & 0.008 \\
\hline Sedentary behaviour (n (\%)) & $1123(55.8)$ & $379(56.8)$ & $235(57.2)$ & $233(63.0)$ & 0.084 \\
\hline BMI (n (\%)) & & & & & $<0.001$ \\
\hline Normal & $1008(50.1)$ & $321(48.1)$ & $170(41.4)$ & $125(33.8)$ & \\
\hline Overweight & $740(36.7)$ & $249(37.3)$ & $170(41.4)$ & $157(42.4)$ & \\
\hline Obese & $266(13.2)$ & $97(14.5)$ & $71(17.3)$ & $88(23.8)$ & \\
\hline Sleep duration (hours/night) $($ mean \pm SD) & $6.9(1.1)$ & $6.9(1.1)$ & $7.1(1.2)$ & $7.1(1.2)$ & 0.010 \\
\hline Hypertension (n (\%)) & $720(35.8)$ & $207(31.1)$ & $181(44)$ & $207(56.0)$ & $<0.001$ \\
\hline Diabetes (n (\%)) & $148(7.4)$ & $52(7.8)$ & $50(12.2)$ & $65(17.6)$ & $<0.001$ \\
\hline Dyslipidaemia (n (\%)) & $286(14.2)$ & $82(12.3)$ & $82(20.0)$ & $102(27.6)$ & $<0.001$ \\
\hline $\begin{array}{l}\text { Excessive daytime sleepiness }(\mathrm{ESS}>10) \dagger \\
(\mathrm{n}(\%))\end{array}$ & $160(8.3)$ & $86(13.5)$ & $56(14.4)$ & $46(13.0)$ & $<0.001$ \\
\hline Severity of sleep apnoeał (n (\%)) & & & & & $<0.001$ \\
\hline Normal & $314(33.1)$ & $103(31.6)$ & $50(26.0)$ & $32(16.8)$ & \\
\hline Mild & $333(35.1)$ & $122(37.4)$ & 76 (39.6) & $60(31.4)$ & \\
\hline Moderate & $193(20.3)$ & $67(20.6)$ & $31(16.2)$ & $58(30.4)$ & \\
\hline Severe & $110(11.6)$ & $34(10.4)$ & $35(18.2)$ & $41(21.5)$ & \\
\hline Depressive status & $190(10.1)$ & $84(13.4)$ & $55(14.4)$ & $75(22.3)$ & $<0.001$ \\
\hline
\end{tabular}

$P$ values from Pearson $\chi^{2}$ or ANOVA where appropriate.

$\mathrm{tn}=3300$.

$\neq \mathrm{n}=1659$. Normal: apnoea-hypopnoea index (AHI) <5/hour; mild: AHI between 5 and 15/hour; moderate: between 15 and 30/hour; severe: >30/hour.

ESS, Epworth sleepiness scale. 
Table 2 Bivariate association between nap frequency and incident cardiovascular disease events, CoLaus study, Lausanne, Switzerland $(n=3462)$

\begin{tabular}{|c|c|c|c|c|c|}
\hline & \multirow[b]{2}{*}{ No nap } & \multicolumn{3}{|c|}{ Nap frequency } & \multirow[b]{2}{*}{$p$-value } \\
\hline & & 1-2 weekly & 3-5 weekly & 6-7 weekly & \\
\hline CVD event & & & & & $<0.001$ \\
\hline $\begin{array}{l}\text { No }(n=3307) \\
(n(\%))\end{array}$ & 1921 (95.4) & $655(98.2)$ & $389(94.7)$ & 342 (92.4) & \\
\hline $\begin{array}{l}\text { Yes }(n=155) \\
(n(\%))\end{array}$ & $93(4.6)$ & $12(1.8)$ & $22(5.4)$ & $28(7.6)$ & \\
\hline
\end{tabular}

P value from Pearson $\chi^{2}$.

CVD, cardiovascular disease.

found between daily nap duration over a week and incidence of CVD events (table 3 ).

The association between taking 1-2 naps weekly and lower incidence of CVD events remained after additionally adjusting for excessive daytime sleepiness $(0.46,0.24-0.89)$ and depressive status $(0.46,0.24-0.90)$, as well as in subjects with at least 6 hours of sleep duration $(0.51,0.26-0.99)$, but not after excluding subjects $<65$ years $(0.82,0.37-1.83)$ or after adjusting for severity of OSA $(0.47,0.20-1.10)$ (see online supplementary table 3). Finally, no association was found between daily nap duration over a week and incidence of CVD events in this sensitivity analysis (see online supplementary table 3 ).

\section{DISCUSSION}

To our knowledge, this is the first population based cohort study investigating the effect of nap frequency and daily nap duration over a week on fatal and non-fatal CVD events. We found that subjects napping once or twice weekly had a lower risk of developing a CVD event compared with non-nappers.

\section{Nap frequency and incidence of CVD events}

Subjects napping once or twice weekly had a lower risk of developing any CVD event compared with non-nappers. This finding is comparable with the result of the Greek cohort study taking nap frequency into account, as they reported lower coronary mortality for subjects napping once or twice weekly compared with non-nappers. ${ }^{4}$ The other study taking nap frequency into account did not differentiate between napping 1-2 times and 3-5 times per week and found no effect of irregular nappers ( $\leq 5$ times weekly) on CVD mortality. ${ }^{9}$ Although taking 1-2 naps weekly was no longer associated with CVD events in some sensitivity analyses ( $>65$ years old or adjustment for OSA), this was most likely due to the low number of subjects included in these analyses ( $n=903$ and $n=1659$, respectively): this led to relatively wide CIs while the effect size remained stable when adjusting for OSA. Another possible explanations is that older nappers do not have time constraints regarding napping and thus take longer naps, which have previously been associated with CVD. ${ }^{11} 13$ Further, older nappers have more comorbidities and the negative association between occasional naps and CVD might be weaker in this population.

In the crude model, we observed a J curved relationship between nap frequency and incidence of CVD events. Interestingly, this finding is in line with previous studies reporting a J curved relationship between nap duration and $\mathrm{CVD}^{11}$ and cardiovascular risk factors, such as diabetes and metabolic syndrome. ${ }^{12}$ However, the increased risk of CVD events for frequent nappers disappeared in adjusted analyses. Regarding the underlying causes of our findings, we could speculate that frequent napping may be secondary to impaired sleep quality due to a chronic condition, which may represent an independent risk factor for CVD events. In contrast, occasional napping might be a result of a physiological compensation allowing for a decrease in stress due to insufficient nocturnal sleep and thus could have a beneficial effect on CVD events. Although the blood pressure and heart rate surge following awakening after an afternoon naps might increase cardiovascular risk in the short term, ${ }^{25}$ the stress releasing result of occasional naps ${ }^{26}$ might counteract this effect and explain the lower risk of CVD events for occasional nappers compared with non-nappers. Interestingly, the beneficial effect of napping once or twice weekly on CVD remained even after controlling for major cardiovascular risk factors, OSA or excessive daytime sleepiness, confounders other studies did not control for.

\section{Nap duration and incidence of CVD events}

We found no association between daily nap duration over a week and incident CVD events. Our findings are partly in line with a meta-analysis, which found no association between short naps $(<1$ hour) and CVD, but contradict their finding that long naps ( $\geq 1$ hour) were positively associated with CVD. ${ }^{11}$ Interestingly, they found a significant $\mathrm{J}$ curved dose-response relationship between nap duration and $\mathrm{CVD}^{11}$; the CVD risk decreased for napping 0-30 $\mathrm{min} /$ day, slightly increased for $45 \mathrm{~min} /$ day napping

Table 3 Effect of nap frequency and duration on incident cardiovascular disease events, CoLaus study, Lausanne, Switzerland ( $\mathrm{n=3462)}$

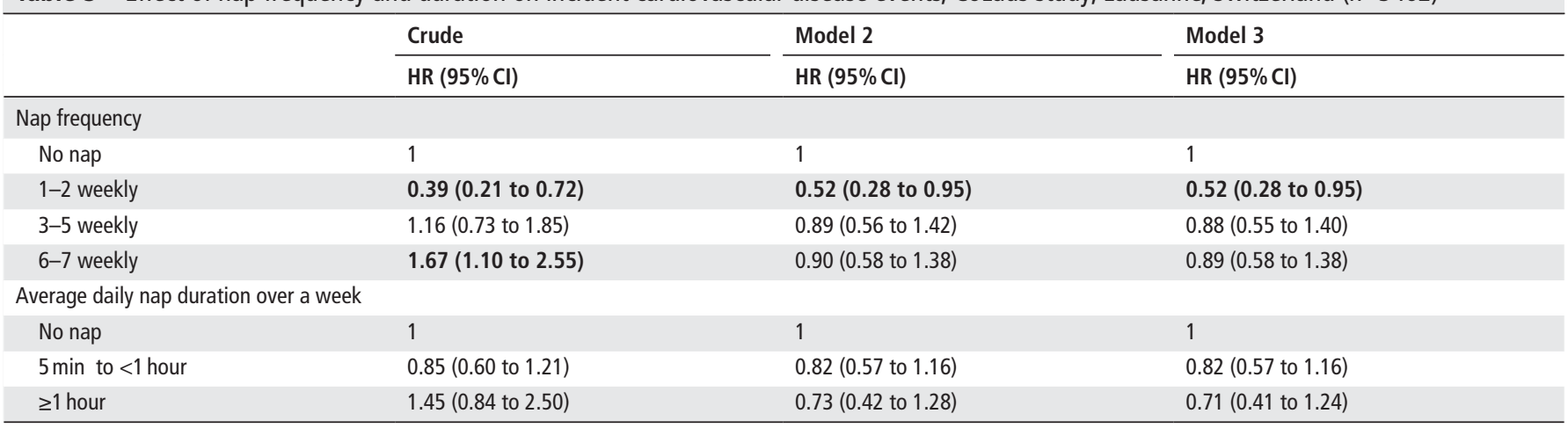

Results are expressed as Cox regression derived HRs $(95 \% \mathrm{Cl})$.

Significant $(\mathrm{P}<0.05)$ associations are indicated in bold type.

Model 2: adjusting for age, sex, education (low, middle, high), smoking status (current, former, never), sedentary behaviour (yes/no), BMI (normal, overweight, obese) and sleep duration (continuous).

Model 3: model 2+adjusting for hypertension, diabetes and dyslipidaemia (categorised as yes/no). 


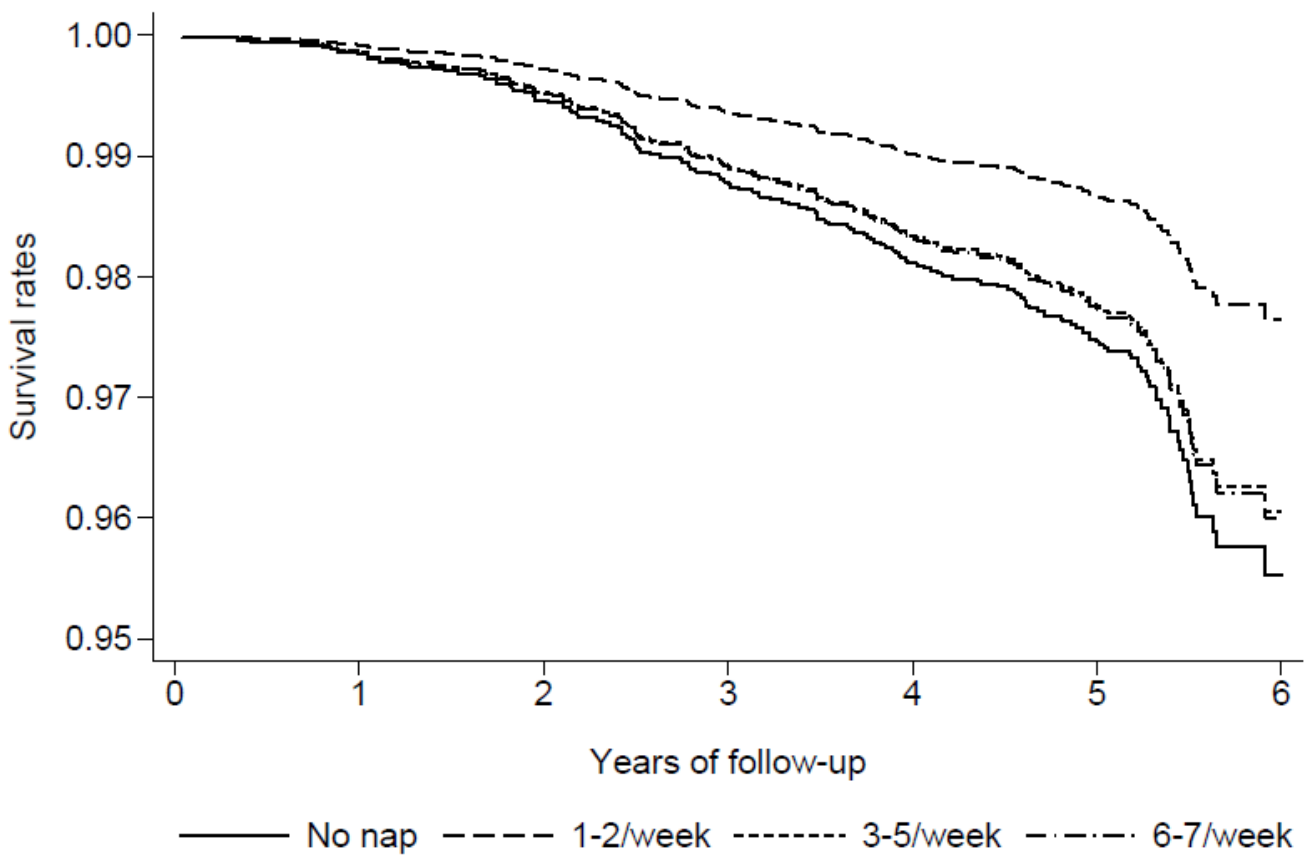

Figure 2 Survival curve, according to nap frequency.

followed by a sharp increase for longer nap durations. Also, our findings are not in line with a previous German study reporting an increased risk of coronary artery disease among subjects taking long naps ( $>1$ hour) at least five times per week. ${ }^{9}$ Further, our findings do not replicate those of previous studies reporting either an adverse dose-response effect of nap duration on $\mathrm{CVD}^{13}$ or a protective dose-response effect of nap duration on coronary mortality. ${ }^{4}$ However, these studies did not control for important confounders, such as physical activity, ${ }^{9} 13$ sleep duration ${ }^{4}$ and major cardiovascular risk factors. ${ }^{4}$ Further, the meta-analysis included heterogeneous studies regarding confounders. ${ }^{11}$ Also,

\section{Key questions}

What is already known about this subject?

- Controversial results regarding the effect of napping on incident cardiovascular events exits. Most studies compare nappers to non-nappers or study nap duration but neglect to take the frequency of napping into account.

What does this study add?

- We found a J curved relationship between nap frequency and cardiovascular events. Whereas the increased risk for frequent nappers disappeared with the inclusion of sociodemographic, lifestyle and cardiovascular risk factors, the negative association between napping once or twice a week and cardiovascular events remained.

\section{How might this impact on clinical practice?}

- Subjects who nap once or twice per week have a lower risk for incident CVD events, while no association was found for more frequent napping or napping duration. Nap frequency may help explain the discrepant findings regarding the association between napping and CVD events. publication bias might exist, leading to an overestimation of an effect. $^{28}$

\section{Strengths and limitations}

Among the strengths of the present study were the population based cohort design and the adjustment for recognised confounding variables, including polysomnography assessed OSA and excessive daytime sleepiness.

We have to acknowledge limitations regarding the reliance of self-reported nap frequency and duration, and the relatively low number of subjects included in our sensitivity analyses. The original question considered napping in the afternoon, so morning naps might have been underestimated. Further, the low number of CVD events in the 1-2 weekly nap group suggests that these results should be interpreted with caution, although Cox models are valid when investigating few events per predictor variable. ${ }^{29}$ Due to the observational nature of the data, we cannot rule out the possibility of residual confounding. The study was conducted in a Swiss population based study, so generalisability to other settings where naps are either more or less frequent is not guaranteed. Hence it would be important that our findings be replicated in other settings.

\section{CONCLUSION}

Subjects who nap once or twice per week have a lower risk of incident CVD events, while no association was found for more frequent napping or napping duration. Nap frequency may help explain the discrepant findings regarding the association between napping and CVD events.

Contributors NH performed the statistical analyses and wrote the article. PM-V collected the data and revised the article for important intellectual content. RH and $J \mathrm{H}-\mathrm{R}$ devised the study and revised the article for important intellectual content. PM-V has full access to the data and is the guarantor of the study.

Funding The CoLaus study was and is supported by research grants from GlaxoSmithKline, the Faculty of Biology and Medicine of Lausanne and the Swiss 
National Science Foundation (grant Nos 33CSCO- 122661, 33CS30-139468 and 33CS30-148401). PM-V received a grant from the Swiss National Science Foundation (320030E_176280) to analyse the associations between sleep and cardiovascular disease

Competing interests PM-V received a grant from the Swiss National Science Foundation (320030E_176280) to analyse the associations between sleep and cardiovascular disease.

Patient consent for publication Not required.

Ethics approval The institutional ethics committee of the University of Lausanne, which afterwards became the Ethics Commission of Canton Vaud (www.cer-vd. ch) approved the CoLaus study (reference 16/03, decisions on 13 January and 10 February 2003) and the approval was renewed for the follow-ups (reference 33/09, decision 23 February 2009; reference 26/14, decision 11 March 2014). The study complies with the Declaration of Helsinki and written informed consent was obtained from all participants.

Provenance and peer review Not commissioned; externally peer reviewed.

Data sharing statement Due to the sensitivity of the data and the lack of consent for online posting, individual data cannot be made accessible. Only metadata will be made available in digital repositories. Metadata requests can also be performed via the study website www.colaus-psycolaus.ch.

ORCID iD

Nadine Häusler http://orcid.org/0000-0003-2389-9365

\section{REFERENCES}

1 Zhong G, Wang Y, Tao T, et al. Daytime napping and mortality from all causes, cardiovascular disease, and cancer: a meta-analysis of prospective cohort studies. Sleep Med 2015:16:811-9.

2 Liu X, Zhang Q, Shang X. Meta-analysis of self-reported daytime napping and risk of cardiovascular or all-cause mortality. Med Sci Monit 2015;21:1269-75.

3 Leng Y, Wainwright NW, Cappuccio FP, et al. Daytime napping and the risk of allcause and cause-specific mortality: a 13-year follow-up of a British population. Am J Epidemiol 2014;179:1115-24.

4 Naska A, Oikonomou E, Trichopoulou A, et al. Siesta in healthy adults and coronary mortality in the general population. Arch Intern Med 2007;167:296-301.

5 Trichopoulos D, Tzonou A, Christopoulos C, et al. Siesta and risk of coronary heart disease. Stress Med 1988:4:143-8.

6 Campos $H$, Siles X. Siesta and the risk of coronary heart disease: results from a population-based, case-control study in Costa Rica. Int J Epidemiol 2000;29:429-37.

7 Stone KL, Ewing SK, Ancoli-Israel S, et al. Self-reported sleep and nap habits and risk of mortality in a large cohort of older women. J Am Geriatr Soc 2009;57:604-11.

8 Tanabe N, Iso H, Seki N, et al. Daytime napping and mortality, with a special reference to cardiovascular disease: the JACC study. Int J Epidemiol 2010;39:233-43.

9 Stang A, Dragano N, Moebus S, et al. Midday naps and the risk of coronary artery disease: results of the Heinz Nixdorf Recall Study. Sleep 2012:35:1705-12.
10 Burazeri G, Gofin J, Kark JD. Siesta and mortality in a Mediterranean population: a community study in Jerusalem. Sleep 2003;26:578-84.

11 Yamada T, Hara K, Shojima N, et al. Daytime napping and the risk of cardiovascular disease and all-cause mortality: a prospective study and dose-response meta-analysis. Sleep 2015;38:1945-53.

12 Yamada T, Shojima N, Yamauchi T, et al. J-curve relation between daytime nap duration and type 2 diabetes or metabolic syndrome: A dose-response meta-analysis. Sci Rep 2016:6:1-10.

13 Wang C, Bangdiwala SI, Rangarajan S, et al. Association of estimated sleep duration and naps with mortality and cardiovascular events: a study of 116632 people from 21 countries. Eur Heart J 2019:40:1-10.

14 Firmann M, Mayor V, Vidal PM, et al. The CoLaus study: a population-based study to investigate the epidemiology and genetic determinants of cardiovascular risk factors and metabolic syndrome. BMC Cardiovasc Disord 2008;8:1-11.

15 Buysse DJ, Reynolds CF, Monk TH, et al. The Pittsburgh Sleep Quality Index: a new instrument for psychiatric practice and research. Psychiatry Res 1989;28:193-213.

16 Johns MW. A new method for measuring daytime sleepiness: The Epworth Sleepiness Scale. Sleep 1991;14:540-5.

17 Heinzer R, Vat S, Marques-Vidal P, et al. Prevalence of sleep-disordered breathing in the general population: the HypnoLaus study. Lancet Respir Med 2015;3:310-8.

18 El Assaad MA, Topouchian JA, Darné BM, et al. Validation of the Omron HEM-907 device for blood pressure measurement. Blood Press Monit 2002;7:237-41.

19 Fuhrer R, Rouillon F. La version francaise de l'echelle CES-D (Center for Epidemiologic Studies-Depression Scale). Description et traduction de l'echelle d'autoévaluation. Psychiatr Psychobiol 1989;4:163-6.

20 Bernstein M, Sloutskis D, Kumanyika S, et al. Data-based approach for developing a physical activity frequency questionnaire. Am J Epidemiol 1998;147:147-54.

21 Bernstein MS, Morabia A, Sloutskis D. Definition and prevalence of sedentarism in an urban population. Am J Public Health 1999;89:862-7.

22 Furihata R, Kaneita Y, Jike M, et al. Napping and associated factors: a Japanese nationwide general population survey. Sleep Med 2016;20:72-9.

23 Empana JP, Dauvilliers Y, Dartigues JF, et al. Excessive daytime sleepiness is an independent risk indicator for cardiovascular mortality in community-dwelling elderly: the three city study. Stroke 2009;40:1219-24.

24 Lasserre AM, Marti-Soler H, Strippoli MP, et al. Clinical and course characteristics of depression and all-cause mortality: A prospective population-based study. J Affect Disord 2016;189:17-24.

25 Bursztyn M, Mekler J, Ben-Ishay D. The siesta and ambulatory blood pressure: is waking up the same in the morning and afternoon? J Hum Hypertens 1996;10:28792.

26 Ramachandruni S, Handberg E, Sheps DS. Acute and chronic psychological stress in coronary disease. Curr Opin Cardiol 2004;19:494-9.

27 Leor J, Poole WK, Kloner RA. Sudden cardiac death triggered by an earthquake. N Engl J Med 1996:334:413-9.

28 Montori VM, Smieja M, Guyatt GH. Publication bias: a brief review for clinicians. Mayo Clin Proc 2000;75:1284-8.

29 Vittinghoff E, McCulloch CE. Relaxing the rule of ten events per variable in logistic and Cox regression. Am J Epidemiol 2007:165:710-8. 